\title{
Recent QCD results on the strange hadron systems
}

\author{
Makoto Oka* \\ Department of Physics, Tokyo Institute of Technology, \\ Meguro, Tokyo, 152-8551, JAPAN \\ E-mail: oka@th.phys.titech.ac.jp
}

\section{Guray Erkol}

Ozyegin University, Kusbakisi Cad. No:2 Altunizade Uskudar Istanbul, 34662 Turkey

E-mail: guray.erkoleozyegin.edu.tr

\section{Toru T. Takahashi}

Yukawa Institute for Theoretical Physics, Kyoto University, Sakyo, Kyoto 606-8502, Japan

E-mail: ttorueyukawa.kyoto-u.ac.jp

\begin{abstract}
Strangeness plays a key role in revealing the nonperturbative properties of QCD in hadron dynamics. The QCD sum rules (QCDSR) and the lattice QCD (LQCD) are applied to understand the most fundamental hadronic interactions, i.e. the couplings of mesons and baryons. The analyses both in QCDSR and LQCD tell us that the SU(3) flavor symmetry, which is an approximate symmetry of QCD, is in fact rather good in the pseudo-scalar-octet-baryon couplings at the physical mass points. We also find that the $F / D$ ratio is close to the value expected from the spin-flavor $\mathrm{SU}(6)$ symmetry. The coupling form factors are also obtained from the lattice data.
\end{abstract}

6th International Workshop on Chiral Dynamics, CD09

July 6-10, 2009

Bern, Switzerland

\footnotetext{
* Speaker.
} 


\section{Introduction}

The QCD lagrangian,

$$
\mathscr{L}=\sum_{q} \bar{\psi}(x)\left(i \gamma^{\mu} D_{\mu}-m_{q}\right) \psi(x)-\frac{1}{4} \sum_{a}\left(G_{\mu \nu}^{a}\right)^{2}
$$

has the exact $\mathrm{SU}\left(\mathrm{N}_{f}\right)$ symmetry, if the quark masses of the $\mathrm{N}_{f}$ flavors are equal. In reality, the quark masses are distributed from a few $\mathrm{MeV}$ to $170 \mathrm{GeV}$ as is shown in Fig. 1. The masses of the "light" quarks, $u$ and $d$, are much smaller than the QCD energy scale $\Lambda_{Q C D}$, and their interactions are constrained by the chiral symmetry that is exact for massless quarks. On the other hand, the "heavy" quarks, $c, b$ and $t$, are much heavier than $\Lambda_{Q C D}$ and therefore heavy quark symmetry can be applied. The strange quark, $s$, has the mass just about $\Lambda_{Q C D}$ and neither the chiral symmetry nor heavy quark symmetry is good enough to neglect symmetry breaking corrections. Thus, the dynamics of the strange quark is most sensitive to the dynamics of QCD.

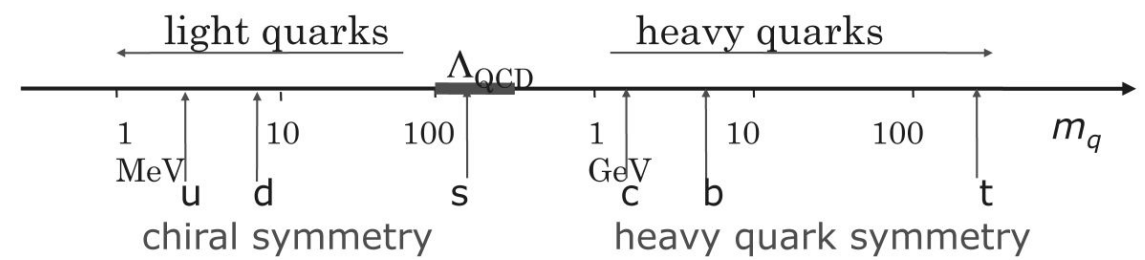

Figure 1: Quark masses and QCD scales.

The quark model with $\mathrm{SU}(3) / \mathrm{SU}(6)$ flavor symmetry has played a major role in the history of hadron physics, but its limitation has become clear in the spectroscopy of excited and exotic hadrons. Indeed, accumulated evidences of new hadrons, such as $\Theta^{+}, X(3872), Z^{+}(4430), \ldots$, have shown that there may exist "sharp" exotic resonances, whose main component is not simply $q q q$ or $q \bar{q}$. It is interesting and important to reveal the dynamics of these exotic states from the QCD point of view. Beyond the quark models, many refined effective models have been proposed to describe hadron spectra and hadron structures, but the predictability of the models is limited as they involve many unknown parameters.

The hadronic interaction is another major subject of the nonperturbative properties of QCD. The nuclear force, among them, is well established and is described by the meson exchange force at long distances with phenomenological short-range interactions. Therefore in order to understand the nuclear force, we need to predict the meson-baryon coupling constants as well as their form factors at the vertex. Again the SU(3) symmetry plays a major role in determining the couplings, while no direct QCD predictions were available.

Recent technical developments in the direct applications of QCD to the spectroscopy start to allow reliable predictions to the hadronic observables from the QCD Lagrangian. In this talk, we present some results of our recent works in which we apply the QCD sum rules (QCDSR) and the lattice QCD (LQCD) to the meson-baryon coupling strengths. ${ }^{1}$

\footnotetext{
${ }^{1}$ The following presentation by P. Gubler at the same conference has discussed the results of the QCDSR for the pentaquark spectrum in detail. [1]
} 


\section{Meson-Baryon Couplings in QCD}

\subsection{SU(3) symmetry of the meson-baryon vertices}

One of the important goals of hypernuclear physics is to study the universal properties of hyperon-nucleon and hyperon-hyperon forces, i.e., generalized nuclear force. Understanding generalized nuclear force from the first principle, QCD, is a key to disentangle origins and mechanisms of hadronic interactions. In particular, the coupling constants of the octet pseudo-scalar mesons $(\pi$, $K, \eta)$ and the octet baryons, $(N, \Lambda, \Sigma, \Xi)$ are the most fundamental quantities in such studies, as the long distance parts of the baryonic interactions in (hyper-)nuclei and nuclear matter are determined by the one-meson-exchange interaction.

In the SU(3) symmetry representations, two octets can be combined into

$$
\mathbf{8} \times \mathbf{8}=\mathbf{1}+\mathbf{8}_{\mathrm{D}}+\mathbf{8}_{\mathrm{F}}+\mathbf{1 0}+\overline{\mathbf{1 0}}+\mathbf{2 7} .
$$

Corresponding to the two octets in the right hand side, two independent coupling constants, $F$ (antisymmetric) and $D$ (symmetric), can be defined. The ratio of the $F$ and $D$ couplings, or $\alpha \equiv$ $F /(F+D)$ is a free parameter in $\mathrm{SU}(3)$.

If the spin-flavor $\mathrm{SU}(6)$ symmetry of the non-relativistic quark model is a good symmetry, then the $F / D$ ratio is fixed to $2 / 3$, or $\alpha=2 / 5$. However, QCD does not necessarily respect the SU(6) symmetry as is seen from the mass splitting of the $N(J=1 / 2)$ and $\Delta(J=3 / 2)$, which belong to the same SU(6) multiplet, 56.

Phenomenological nuclear potential models often assume the SU(3) invariance of the meson baryon coupling constants. They assume that the $F / D$ ratio is one of the fitting parameters. In the following, we apply the QCD sum rule method and lattice QCD to the meson-baryon coupling constants and see what QCD predicts for the $\mathrm{SU}(3)$ invariance and the $F / D$ ratios.

\subsection{QCD sum rules for meson-baryon coupling constants}

Doi et al. constructed the QCD sum rules for the coupling constants of the octet pseudoscalar mesons and octet baryons [3]. The two-point correlation functions with the pion in the initial state are analyzed in the QCD sum rule techniques. It is found that the validity of the sum rules depend on the Dirac-matrix structure of the correlators, and that the tensor structure (proportional to $\sigma_{\mu \nu}$ ) gives the most reliable sum rules. In particular, the dependence on the choice of the baryon operators is minimal for the tensor sum rules. We have evaluated the $F / D$ ratio in the SU(3) limit (and also in the chiral limit) of the sum rule. The obtained ratio is $F / D=0.65 \pm 0.10$, which is very close to the $\mathrm{SU}(6)$ value, $2 / 3$, and also consistent with the value, $F / D=0.57$, of the axial-vector coupling constants, $g_{A}$, determined by the beta decays of octet baryons. We also find that the $\mathrm{SU}(3)$ symmetry relations are fairly well satisfied for the $\pi B B^{\prime}$ coupling constants.

The most reliable estimate of the absolute value of the $\pi N N$ coupling is given by the projected correlated function method by Kondo et al. [4] They gave $g_{\pi N N}=9.6 \pm 1.6$, which is slightly smaller than the empirical value, $g_{\pi N N} \sim 12.8$.

The couplings of the scalar mesons: $\sigma, a_{0}, f_{0}$ and $\kappa$, have been studied in the QCD sum rules [5], resulting in the $\alpha \sim 0.55$, but significant SU(3) breaking effects are observed. Both the $\Sigma-\sigma$ and $\Xi-\sigma$ couplings are seen to be enhanced by $30-50 \%$ from those in the SU(3) limit. It is 
also found that the baryon-scalar-meson coupling strengths can be a good indicator of the 4-quark structure of the scalar mesons.

\subsection{Lattice QCD simulation of the meson-baryon couplings}

We have performed a full-QCD lattice simulation of the three-point functions [6], using the CP-PACS gauge configurations with 2-flavor dynamical quarks on the $16^{3} \times 32$ lattice. [2] They are generated by using the renormalization-group-improved gauge action and the mean-field improved clover quark action. The lattice spacing corresponds to $a=0.1555 \mathrm{fm}$, or $a^{-1}=1.267 \mathrm{GeV}$ for $\beta=1.95$ ). The ratio and absolute values of the coupling constants are obtained for several quark masses: $m_{q} \sim 150,100,65$, and $35 \mathrm{MeV}$.

Three point correlation function

$$
\left\langle G^{\mathscr{B} \mathscr{P} \mathscr{B}^{\prime}}\left(t_{2}, t_{1} ; \mathbf{p}^{\prime}, \mathbf{p} ; \Gamma\right)\right\rangle=-i \sum_{\mathbf{x}_{2}, \mathbf{x}_{1}} e^{-i \mathbf{p} \cdot \mathbf{x}_{2}} e^{i \mathbf{q} \cdot \mathbf{x}_{1}} \Gamma^{\alpha \alpha^{\prime}}\left\langle\operatorname{vac}\left|T\left[\eta_{\mathscr{B}}^{\alpha}\left(x_{2}\right) P\left(x_{1}\right) \bar{\eta}_{\mathscr{B}^{\prime}}^{\alpha^{\prime}}(0)\right]\right| \operatorname{vac}\right\rangle,
$$

is obtained in the simulation, which gives the desired pseudoscalar form factor when $t_{2}-t_{1}$ and $t_{1}$ are large. The form factor can be fitted very well with the monopole form:

$$
g_{M B B^{\prime}}\left(q^{2}\right)=g_{M B B^{\prime}} \frac{\Lambda_{M B B^{\prime}}^{2}}{\Lambda_{M B B^{\prime}}^{2}-q^{2}} .
$$

Fig. 2 shows the $\pi N N$ coupling constants and the corresponding monopole cut-off mass $\Lambda_{M B B^{\prime}}$. It is found that the $\pi N N$ coupling constants have little dependence on the light $(u, d)$ quark mass, and that the obtained value of the $\pi N N$ coupling constant is consistent with the phenomenological value used in the meson exchange $N N$ potential models, $g_{\pi N N}$ (pheno.) $\sim 12.8$. On the other hand, the monopole cut-off mass is obtained to be $\Lambda_{\pi N N} \sim 0.7-0.8 \mathrm{GeV}$, which is softer than the one used in the meson exchange models. These results are consistent with the QCDSR.

The open circles in Fig. 2 shows the $\mathrm{SU}(3)$ limit, where the $u, d$ quark masses are equal to the (valence) $s$ quark, ${ }^{2}$ which is fixed in these calculations. We have derived the $\alpha=F /(F+D)$ ratio at this $\mathrm{SU}(3)$ limit point and obtain $\alpha=0.395(6)$. It is quite interesting that the values is close to the spin-flavor SU(6) limit, or the naive quark model results, which is not a symmetry of QCD. The SU(6) symmetry has been used often to understand the structures of the low-lying mesons and baryons, in particular, the magnetic moments and other electro-magnetic properties of baryons. The symmetry is, however, not well founded in the QCD, as it is valid for non-relativistic quarks. Nevertheless, we here find that the couplings of the pseudo-scalar meson and octet baryon agree with the SU(6) prediction. It is very interesting to see whether this is accidental or not.

Our results show further surprise that even when the mass differences between the $u, d$ and $s$ quarks are turned on, the SU(3) symmetry is kept rather well. Fig. 3 shows the ratios of the various coupling constants to the $\pi N N$ coupling. They show mild quark mass dependences, but the results are rather stable. By comparing these ratios to the $\mathrm{SU}(3)$ relations, we find that the violation o $\mathrm{SU}(3)$ is less than $10 \%$. (See [6] for the definitions of the SU(3) breaking and further details.) In Fig. 4 , the $\alpha$ ratio obtained by the global fit of the SU(3) relations for all the quark masses are

\footnotetext{
${ }^{2}$ Note that the gauge configurations employed here are generated with two light quarks, so that no strange-quark loops are included in this calculation.
} 


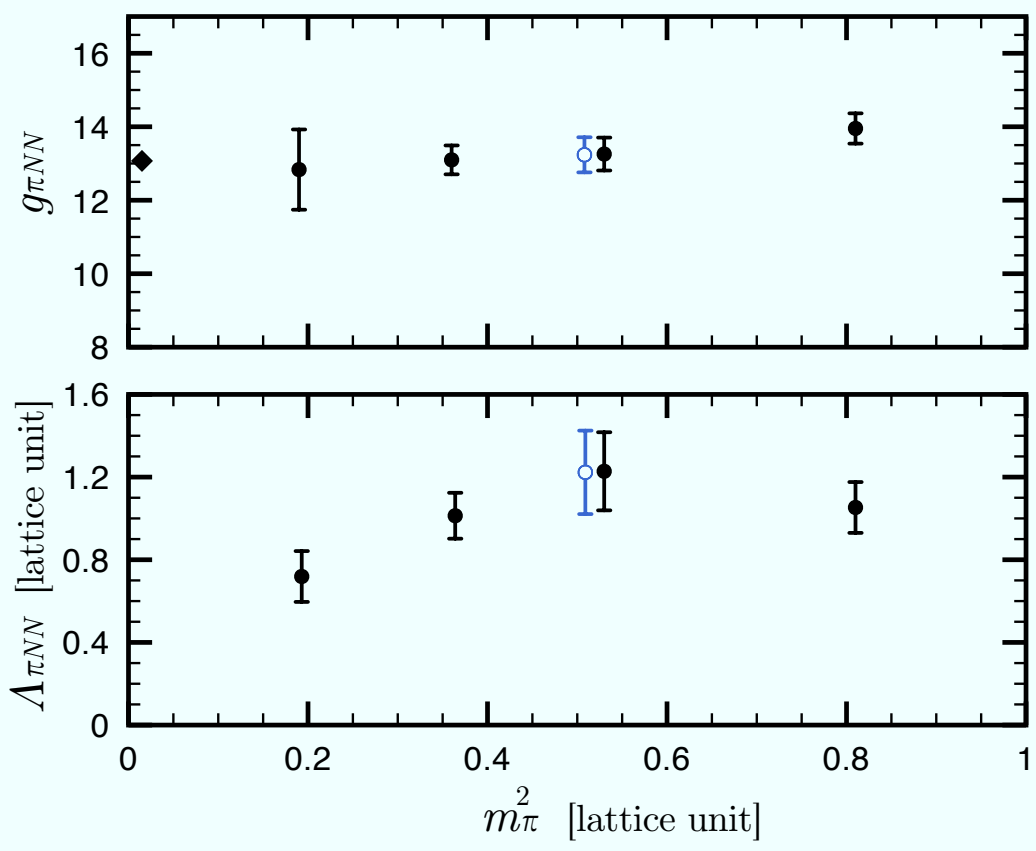

Figure 2: The $\pi N N$ coupling constant $g_{\pi N N}$ and the monopole cutoff $\Lambda$ of the vertex form factor. The open circles show the values where the SU(3) symmetry is satisfied (for valence quarks). The phenomenological value of the coupling constant, $g_{\pi N N} \sim 12.8$ is indicated as the square. The lattice unit is $a^{-1}=1.267 \mathrm{GeV}$.

shown. We find that the $\alpha$ decreases as the quark mass decreases, while the $\mathrm{SU}(3)$ symmetry is kept satisfied.

\section{Conclusion and Outlook}

We have presented the results of the calculation of the coupling constants and the form factors of the pseudo-scalar mesons and the octet baryons using the QCD sum rules and the lattice QCD. It is found that the SU(3) symmetry is fairly good even if the strange quark mass breaks the SU(3) symmetry in the QCD lagrangian. We also find that the $F / D$ ratio is close to that predicted in the spin-flavor SU(6) symmetry. The form factors obtained in the lattice QCD calculation are well fitted by the monopole function, and the cutoff factor $\Lambda$ is obtained by fitting to the lattice data, leading a smaller cutoff compared with the phenomenological meson-exchange potential models.

We stress importance of QCD-based calculations in order to understand the nature of the hadronic interactions as well as the new (exotic) multi-quark world of hadrons. The lattice QCD has reached the position in which the real quark-mass full QCD is accessible. [7] Application of the lattice QCD to hadronic interactions and (exotic) resonance spectra have just begun and will flourish in near future. One essential problem to be solved is how to separate resonances from scattering states. In principle, measuring the phase shifts of relevant hadron-hadron scattering may 

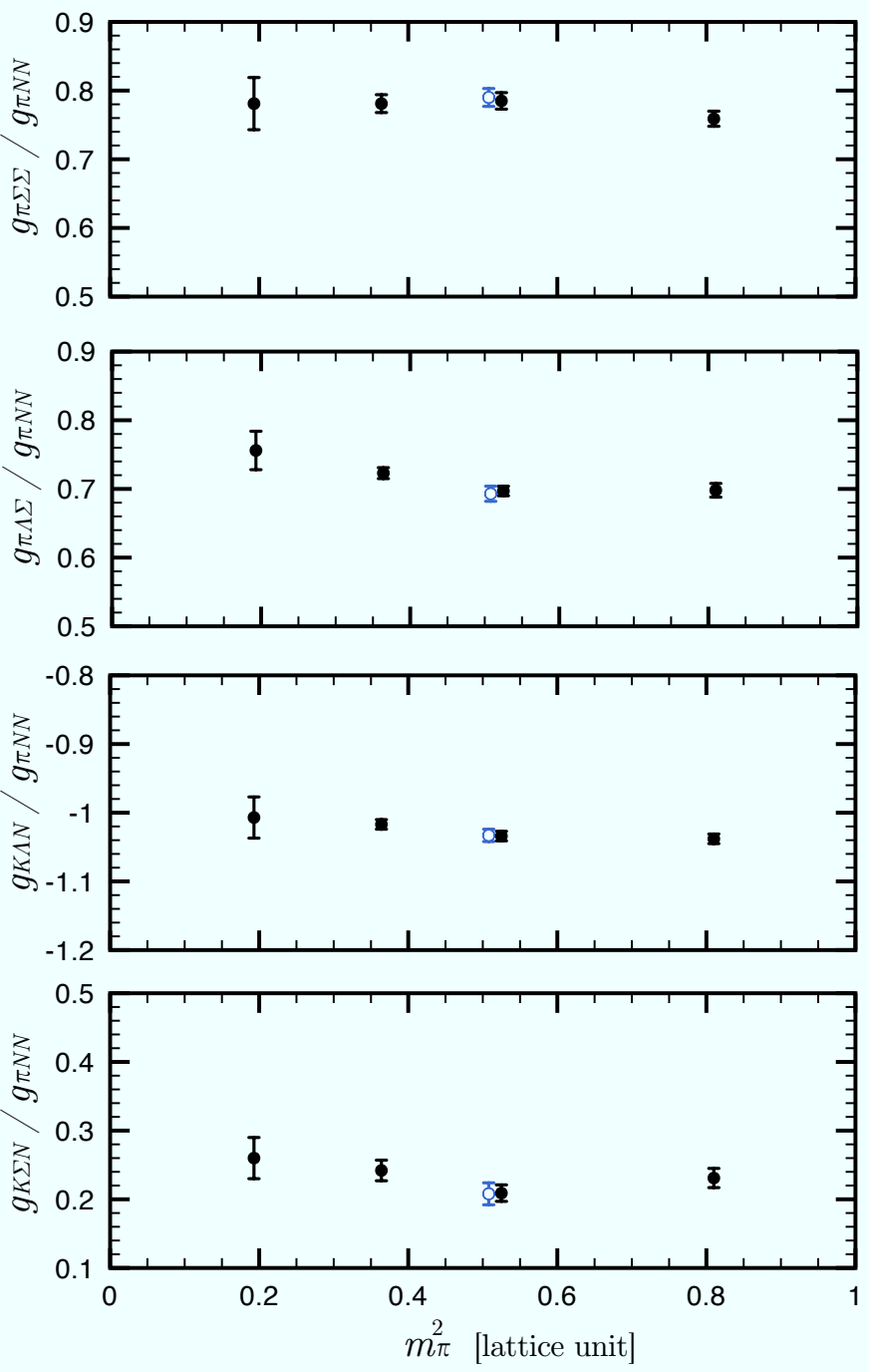

Figure 3: The ratios of the coupling constants of the pseudoscalar mesons and the octet baryons.

give us resonance spectrum, but, in practice, it costs a huge computation. Other methods using for instance dependence on the boundary conditions may be useful.

The strange systems supply many subjects, which are ideal for checking physical pictures of hadron dynamics. Thus, hypernuclear physics as well as kaon and hyperon physics are to be studied more in the future. The JPARC facility, which has just started its operation at Tokai, Japan, is a dream machine for strangeness hadron physics. Further strong collaboration of the theory and experimental researches is anticipated.

\section{Acknowledgments}

This work was supported in part by KAKENHI (17070002 and 19540275). M.O. would like to acknowledge the collaborators whose contributions are presented in this report: Drs. Takumi 


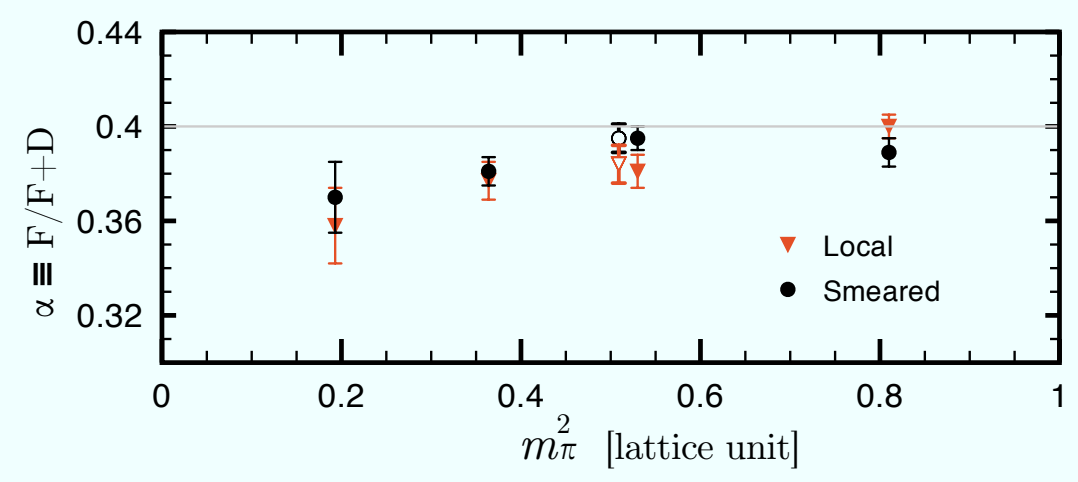

Figure 4: The parameter $\alpha=F /(F+D)$ of the PS meson-baryon couplings obtained by the global fitting of the $\mathrm{SU}(3)$ relations.

Doi, Hung-chong Kim, Yoshihiko Kondo.

\section{References}

[1] P. Gubler, D. Jido, T. Kojo, T. Nishikawa, M. Oka, Phys. Rev. D79 (2009) 114011, arXiv:0902.2049.

[2] CP-PACS Collaboration: A. Ali Khan et al., Phys. Rev. D65 (2002) 054505, Erratum-ibid. D67 (2003) 059901, arXiv:hep-lat/0105015.

[3] T. Doi, H. Kim and M. Oka, Phys. Rev. C62 (2000) 055202; T. Doi, Y. Kondo and M. Oka, Phys. Rept. 398 (2004) 253-279.

[4] Y. Kondo and O. Morimatsu, Nucl. Phys. A717 (2003) 55-72.

[5] G. Erkol, et al., Phys. Rev. C73 (2006) 044009; Phys. Rev. C74 (2006) 045201.

[6] G. Erkol, M. Oka and T.T. Takahashi, Phys. Rev. D79 (2009) 074509, arXiv:0805.3068.

[7] PACS-CS Collaboration: S. Aoki, et al., arXiv:0807.1661v1 [hep-lat]. 